\title{
The Dynamic Interplay between Cell Membranes and Membrane Proteins Rana Ashkar ${ }^{1}$ \\ ${ }^{1}$ Virginia Tech \\ ashkar@vt.edu
}

Biological cell membranes, primarily composed of lipids and cholesterol, harbor a wide diversity of proteins responsible for various cellular processes, including ion gating, signal transduction, and other functions that are central to the cell viability. While it is well recognized that protein functions are facilitated by synergistic interactions with their host membrane, very little is known about the dynamic interplay between proteins and their lipid membrane environment. Interestingly, the energy landscape of membrane proteins shows that conformational changes in proteins - necessary for their regulatory role - happen over time scales commensurate with thermal membrane fluctuations. In membranes, these fluctuations primarily manifest in the form of bending undulations and membrane thickness fluctuations. Therefore, knowing how such fluctuations could influence, or respond to, changes in protein confirmations is key to advancing our fundamental understanding of membranes as dynamic platforms for regulated protein functions and to accelerating informed designs of cellular therapeutics. This talk discusses progress in neutron spin echo spectroscopy, combined with molecular dynamics simulations, in directly interrogating collective fluctuations in model cell membranes and their response to compositional variations, environmental changes, and molecular additives. The talk will emphasize recent studies on the dynamic response of model membranes to conformational changes of a tunable peptide, namely $\mathrm{pH}$ low insertion peptide (pHLIP). The results show that pHLIP has distinct effects on bending and thickness fluctuations in its surface-associated vs transmembrane state, suggesting that modifications in membrane fluctuations could serve as an intermediary mechanism for enabling long-range protein-induced processes. 